\title{
Evaluating the Prognostic Role of Monocytopenia in Chemotherapy-Induced Febrile Neutropenia Patients Treated with Granulocyte Colony-Stimulating Factor
}

\author{
Osama Alshari $\mathbb{D}^{\prime}$ \\ Yazan O Al Zu'bi (D) ${ }^{2}$ \\ Ahmed H Al Sharie $\mathbb{D D}^{2}$ \\ Farouk $\mathrm{H}$ Wafai $\mathbb{D}^{2}$ \\ Abdelwahab J Aleshawi ${ }^{2}$ \\ Farah $\mathrm{H}$ Atawneh ${ }^{3}$ \\ Hasan A Obeidat ${ }^{2}$ \\ Majd N Daoud ${ }^{2}$ \\ Mohammad Z Khrais ${ }^{4}$ \\ Dima Albals ${ }^{5}$ \\ Faize Tubaishat ${ }^{6}$ \\ 'Division of Oncology, Department of \\ Internal Medicine, Faculty of Medicine, \\ Jordan University of Science \& \\ Technology, Irbid, Jordan; ${ }^{2}$ Faculty of \\ Medicine, Jordan University of Science \& \\ Technology, Irbid, Jordan; ${ }^{3}$ Department of \\ Medical Laboratory Sciences, Faculty of \\ Applied Medical Sciences, Jordan \\ University of Science and Technology, \\ Irbid, Jordan; ${ }^{4}$ Department of Internal \\ Medicine, King Hussein Cancer \\ Foundation and Center, Amman, Jordan; \\ ${ }^{5}$ Department of Medicinal Chemistry and \\ Pharmacognosy, Faculty of Pharmacy, \\ Yarmouk University, Irbid, Jordan; \\ ${ }^{6}$ Division of Oncology, Department of \\ Internal Medicine, Al Bashir Hospital, \\ Amman, Jordan
}

\begin{abstract}
Objective: Chemotherapy-induced febrile neutropenia is a common and serious oncological emergency which carries a substantial mortality and morbidity. The main objective of this study is to evaluate the usage of absolute monocyte count (AMC) at presentation as a prognostic factor for patients with chemotherapy-induced febrile neutropenia who were subsequently treated with granulocyte colony-stimulating factor (G-CSF).
\end{abstract}

Study Design: The electronic medical records of our center were used retrospectively to identify patients diagnosed with unprecedented chemotherapy-induced febrile neutropenia treated with G-CSF between January 2010 to December 2020 and diagnosed with solid and hematological malignancies. Patient's demographics, disease characteristics and laboratory investigations were extracted. Disease progression measures were statistically compared between the study groups in the short-term period of follow-up (six days) including absolute neutrophil count (ANC), ANC difference compared to the baseline readings, hospitalization period, and mortality.

Results: A total of 80 patients were identified and categorized into two groups namely monocytopenia $(\mathrm{n}=34)$ and non-monocytopenia $(\mathrm{n}=46)$ with an AMC cutoff point of $0.1 \times 10^{9}$ cells $/ \mathrm{L}$. The monocytopenia group exhibited a worse prognosis with lower ANC values and slower improvement illustrated by the low ANC difference values at all follow up points $(P$-value $\leq 0.05)$ apart from day 5 . A statistically significant lower hospitalization period was also observed in the non-monocytopenia group $(P$-value $=0.006)$. Linear regression analysis evaluated the association between AMC values at admission and ANC values at admission along with subsequent days of follow up which were found to be statistically significant $(P$-value $\leq 0.05)$. Receiver operating characteristic curves suggest a satisfactory predictability of ANC changes by AMC values at admission, days1, 2, 3, 4 and 6 .

Conclusion: Monocytopenia holds a worse prognosis in chemotherapy-induced febrile neutropenia patients treated with G-CSF. In addition, AMC values at presentation represents a potential risk factor that can predict short-term changes regarding ANC measures.

Keywords: febrile neutropenia, granulocyte colony-stimulating factor, G-CSF, chemotherapy, absolute monocyte count, monocytopenia, absolute neutrophil count

\section{Introduction}

Febrile Neutropenia is a major and critical complication of chemotherapy provided for cancer patients, ensuring a tremendous impact on healthcare resources and patients' outcomes. ${ }^{1}$ Neutropenia is defined by the American Society of Clinical Oncology (ASCO) and Infectious Diseases Society of America (IDSA) joint guideline as an absolute neutrophil count (ANC) of less than 1000 cells $/ \mu \mathrm{L}$. Moreover,
Correspondence: Osama Alshari Division of Oncology, Department of Internal Medicine, Faculty of Medicine, University of Science \& Technology, P. O. Box: 3030, Irbid, 22II10, Jordan Email osamasalshari@gmail.com 
fever in neutropenic patients has been defined as a single oral temperature of $\geq 38.3^{\circ} \mathrm{C}$ or a temperature of $\geq 38.0^{\circ} \mathrm{C}$ persisting over 1 hour. $^{2}$ A consistent definition is also provided by the European Society for Medical Oncology (ESMO) clinical practice guidelines, in which febrile neutropenia is defined as an oral temperature of more than $38.3^{\circ} \mathrm{C}$ or two consecutive readings of more than $38.0^{\circ} \mathrm{C}$ for two hours and an ANC of less than $0.5 \times 10^{9} / \mathrm{L}$ or expected to fall below it. ${ }^{3}$

Various pathophysiological pathways have been hypothesized previously to determine the probable cause of fever in neutropenic patients. To begin with, it has been regarded to the cytokines release by the immune cells and stromal cells in response to microorganisms and molecular motifs. ${ }^{4}$ Furthermore, a reduction in anti-inflammatory molecules such as interleukin-10 (IL-10), antimicrobial peptides such as interleukin-1 (IL-1) receptor antagonists would prevent the physiological feedback inhibition of inflammation, intensifying the damage. ${ }^{4}$ An elevation in uric acid levels may have a role as well, amplifying the response of innate immune cells to microbial stimulation by elevating the proinflammatory cytokines; tumor necrosis factor-alpha (TNF- $\alpha$ ) and interleukin-1 alpha (IL-1 $\alpha){ }^{5}$ A vast amount of evidence regards fever to be a consequence of infection since patients are at a higher risk, albeit the lack of microbiological evidence in certain cases. ${ }^{6}$ Fever of unidentified origin was observed in almost $40 \%$ of neutropenic patients. ${ }^{7}$ A potential reason behind such phenomena can be justified by the release of danger-associated molecular patterns (DAMPs) because of mucosal barrier injury due to the cytotoxic effects of chemotherapeutic agents, which along with the pathogen-associated molecular patterns (PAMPs) eventually results in a substantial inflammatory response provoking fever. ${ }^{4}$

The consequences of febrile neutropenia remain a source of concern that cannot be disregarded with major complications effecting the patient's treatment outcomes, cost, morbidity, and mortality, yet, variations exist due to the cancer type and stage, its treatment, and patient's demographics. ${ }^{20}$ The economic burden is considerable with the costs going from $\$ 16,000$ to $\$ 19,000$ for the extraordinary greater part of patients being hospitalized, in which they require a prolonged hospitalization care period, which might end with the patient's death. ${ }^{8,9}$ Additionally, febrile neutropenia patients are 50\% more likely to have an infection with $20 \%$ of the patients having bacteremia. The most common sights infected being the lungs, the gastrointestinal tract, and the skin. ${ }^{22}$ The most common pathogens isolated were gram negative organisms Escherichia coli, Pseudomonas aeruginosa and Klebsiella pneumoniae. However, the trend is changing with the predominance of gram-positive organisms such as methicillin-resistant Staphylococcus aureus (MRSA), as well as a rise of fungal infections, in particular Candida. $^{10}$ Therefore, the cornerstone of treatment for febrile neutropenic patients are antibiotics. Low risk patients are indicated oral antibiotics with fluoroquinolone as a primary treatment, which can be given in an outpatient setting even though patients should be cautiously chosen with attentive monitoring for any emergency if one occurs. In comparison, high risk patients are admitted with an intravenous (IV) antibiotics treatment plan including antipseudomonal beta-lactam agents. ${ }^{1}$ It is recommended that all patients are continued on antibiotics until the patient is afebrile for at least 48 hours with an ANC exceeding 500 cells $/ \mathrm{mm}^{3}$. 1,11 Nonetheless, if the patient does not improve, antimicrobial coverage ought to be expanded to cover resistant gram negative, gram positive, anaerobic microorganisms, and fungi. $^{11}$

Notwithstanding anti-microbials, colony stimulating factors might be an essential part of a treatment plan. Evidence from existing literature has proven the favorable effects of colony stimulating factors in reducing the hospitalization and IV antibiotics administration periods. ${ }^{12}$ Even though G-CSF does not reduce mortality in chemotherapy induced febrile neutropenia, it has been found that G-CSF added to antibiotics reduces the hospitalization period, antibiotic use, fever, neutropenia, and recovery of the neutrophils number duration. ${ }^{13,14}$ Since a growing body of research suggests that the clinical course of neutropenic patients could be predicted by the usage of monocyte measures as monocyte percentage (MP) and absolute monocyte count (AMC). In this work, we hypothesized and tested the idea suggesting that the AMC could predict the clinical responsiveness of G-CSF therapy in febrile neutropenia patients without any age, gender, chemotherapy regimen or malignancy restrictions.

\section{Patients and Methods Patients and Data Collection}

The aim of this study is to evaluate the utilization of AMC at presentation as a prognostic factor for patients with chemotherapy-induced febrile neutropenia and subsequently treated with G-CSF. Data was collected from King Abdullah University Hospital (KAUH), a tertiary 
care center located in Ar Ramtha, Jordan. We retrospectively identified patients diagnosed with chemotherapyinduced febrile neutropenia for the first time and treated with G-CSF according to the current hospital guidelines between January 2010 to December 2020. The hospital electronic medical records were used to extract the following data including demographics, type of malignancy (solid tumors versus hematological malignancies), distant metastasis, bone marrow involvement, chemotherapeutic regimens and its current cycle, temperature at admission, clinical presentation, comorbidities, blood culture results, number of febrile neutropenia attacks, hospitalization period and mortality. Complete blood count (CBC) and the white blood cells (WBC) differential were also extracted at different time intervals representing the short-term progression or improvement after admission and treatment with G-CSF including day 1 to 6 . After day 6, a minority of patients possessed a laboratory follow up results impairing the statistical judgment due to low statistical power, hence they were excluded from the analysis.

A total of 80 patients were enrolled. Exclusion criteria included patients missing key data such as $\mathrm{CBC}$ and WBC differential at presentation with no follow up laboratory results, patients did not get G-CSF therapy, non-malignant cases which developed febrile neutropenia including ChediakHigashi syndrome, aplastic anemia, acquired immunodeficiency syndrome (AIDS), neutropenia, cyclical neutropenia, alcoholic liver cirrhosis and a severe malnutrition case. The first attack was only included in the analysis for patients who developed multiple febrile neutropenia attacks, to eliminate the personalized response bias. Patients were then categorized based on AMC values into two groups namely monocytopenia group $(n=34)$ and a non-monocytopenia group $(n=46)$ with a cutoff point of $0.1 \times 10^{9}$ cells $/ \mathrm{L}$ as previously described. ${ }^{15-19}$ ANC and ANC differences at each follow up interval compared to ANC at admission were calculated. This study was conducted in accordance with the declaration of Helsinki, applying all the current regulations for retrospective studies in KAUH. Patient's consent was waived as data was used in aggregate anonymously.

\section{Statistical Analysis}

Statistical analysis was performed using the IBM SPSS statistical package for Windows v.26 (Armonk, NY, USA). Data was expressed as frequency (percentage) for nominal data, mean \pm standard deviation of the mean (SD) for normally distributed continuous variables or median
(Interquartile range) for non-normally distributed continuous variables. Normality was tested using Shapiro-Wilk test. Statistical significance between the study groups regarding the previously mentioned parameters was determined using Chi-square test or Fisher's exact test, likelihood ratio accordingly, for categorical variables and Mann-Whitney $U$-test for non-normally distributed continuous variables and independent $t$-test for normally distributed continuous variables. $P \leq 0.05$ was considered statistically significant.

A simple linear regression was used to assess the direction and strength of the relationship between the AMC at admission and the ANC at admission and different time intervals of follow up (day 1-6). The data was transformed using the natural logarithm (ln) since the data was skewed to the right. The sensitivity and specificity of AMC values as a predictor variable for the ANC change at different time intervals was assessed using the receiver operating characteristic (ROC) curves. The ANC change at different time intervals was dichotomized into a binary system (1 for an increase and 0 for a decrease or no-change compared to reported admission ANC value) as previously described. ${ }^{20}$ ROC parameters were calculated including the area under the curve (AUC) and the cut-off point which indicates the point of the maximum sensitivity and specificity. The Kaplan-Meier analysis was used to calculate the probability of a second attack and the probability of survival as a function of time. Second attack and death were coded a value of 1 . While patients who lived or did not develop a second attack during the period of data collection were assigned as 0 . Statistical significance between Kaplan-Meier curves were tested using the Log rank test. Graphs were generated using GraphPad Prism 8 (San Diego, CA, USA).

\section{Results}

A total of 80 patients were enrolled in the analysis, ranging in age from 1 to 78 years-old, who were admitted to the hospital for a febrile neutropenia attack (47.5\% males and $52.5 \%$ females). According to the AMC values at admission, patients were divided into two subgroups; 34 patients (42.5\%) were included in the monocytopenia group, while the non-monocytopenia group included 46 patients $(57.5 \%)$. According to the type of malignancy 36 patients $(45 \%)$ had a hematological malignancy in which Hodgkin's lymphoma, non-Hodgkin's lymphoma and diffuse large B-cell lymphoma were the highest three occurring malignancies, on the other hand, breast cancer, neuroblastoma and Ewing's sarcoma were the most 
occurring solid malignancies $(55 \%, \mathrm{n}=44)$. Of the 80 patients included, 12 had a bone marrow involvement, in addition to 17 patients $(21.3 \%)$ who had a distant metastasis. Patient's chemotherapy regimens varied, with R-CHOP (15\%), ABVD (6.3\%), AC (6.3\%), VCD (5\%), and ICE (5\%) being the most used protocols (Supplementary data, Table S1), with a median of 3.00 (3.00) prior cycles of treatment. Additionally, most patients presented with fever solely in both monocytopenia $(61.8 \%, \mathrm{n}=21)$ and non-monocytopenia $(54.3 \%, \mathrm{n}=25)$ groups, succeeded by fever with cough $(12.5 \%, \mathrm{n}=10)$ and fever with general weakness $(7.5 \%, \mathrm{n}=6)$ respectively. At the time of admission, the patients's temperatures ranged between $38^{\circ} \mathrm{C}$ and $41^{\circ} \mathrm{C}$. Principally, patients were free of any co-morbidity, apart from 21 patients who had one or more co-morbidities, with diabetes mellitus and hypertension being the dominant observations. In 10 patients, there was an obvious indication of a microbiologically defined infection, with five having a positive blood culture, four having a positive urine culture, and only one having a positive sputum culture (Supplementary data, Table S2). No statistically significant difference was observed amidst demographics and baseline characteristics, apart from gender $(P$-value $=0.049)$ when comparing the mentioned subgroups (Table 1).

The CBC parameters alongside the WBC differential values were obtained at admission and at different follow up time intervals up to 6 days following the attack. Statistical significance was calculated between the study groups regarding the previously mentioned parameters (Supplementary data, Table S3-S9). At admission, no significance was highlighted between both groups except for baseline WBCs count $(P$-value $<0.001)$, red cell distribution width $(\mathrm{RDW})(P$-value $=0.026)$, platelets count $(P$-value $=0.016)$, lymphocytes $(P$-value $<0.001)$ and basophils $(P$-value $=0.041)$. WBCs count illustrated a significant difference at all the observational readings apart from day five, in which the non-monocytopenia group exhibited a better prognosis presented by a higher WBCs count at follow ups. The same trend was also seen in the neutrophil's percentage and ANC values, emphasizing the importance of monocytopenia in determining the improvement degree of febrile neutropenia patients after G-CSF therapy. The statistically significant difference observed regarding other $\mathrm{CBC}$ parameters and WBC differential values at different follow ups will not be discussed since they are totally not germane. But such analysis was performed to explore the possible concurrent significant variables that could be optimized and utilized as predictive measures for future work as in the case of lymphocyte percentage. Additional measures including liver and kidney function tests were also shown for both groups (Supplementary data, Table S10 and S11).

The main prognostic outcomes included in the analysis were WBCs count, neutrophils percentage, ANC, ANC difference at different follow up time intervals, and the hospitalization period. ANC difference represent the degree of improvement calculated by the mathematical difference in ANC at a specific follow up point compared to the baseline reading. Albeit the lack of significance identified in neutrophils percentage and ANC at admission, they have showed to be significant at all the follow up intervals as illustrated in Table 2. Figure 1 illustrates a visual representation of the ANC values at admission and different follow up intervals regarding the monocytopenia and nonmonocytopenia groups, which shows a preferred prognostic path displayed by the non-monocytopenia group with a curve up shift to a higher ANC values, thus a better response to G-CSF treatment. ANC difference was also significant at all the follow up observations. Initially, on day one $(P$-value $=0.006)$ and subsequently progressing through the consecutive days, day two ( $P$-value $<$ $0.001)$, day three $(P$-value $<0.001)$, day four $(P$-value $=$ $0.001)$, and day six $(P$-value $=0.019)$, except day five with $P$-value of 0.056 . The hospitalization period of patients within the monocytopenia group was 6.0 (2.25) which was higher compared to the non-monocytopenia group which was 5.0 (3.00) $(P$-value $=0.006)$.

As a result of the different hospitalization periods, 67 patients were tested on the first day of follow up, 78 in the second, 64 on the third, 46 on the fourth, 32 on the fifth, and only 20 on the sixth day out of the 80 patients included in the present study which subsequently conducted a CBC test on the day of admission. To evaluate the relationship between $\mathrm{AMC}$ values at admission and $\mathrm{ANC}$ at different time intervals, linear regression analysis was utilized to further examine this relationship, since ANC difference at different time intervals exhibited a significant difference between monocytopenia and nonmonocytopenia groups. The AMC values at admission were found to have a significantly positive relationship with ANC levels at various time intervals (Figure 2). A significant linear equation was found for day one $(\mathrm{F}$ $(1,65)=43.943, P$-value $\left.<0.001, \mathrm{R}^{2}=0.403\right)(\beta=0.736$, $P$-value $<0.001)$, day two $(\mathrm{F}(1,76)=112.744, P$-value $<$ $\left.0.001, \mathrm{R}^{2}=0.597\right)(\beta=1.049, P$-value $<0.001)$, day three 
Table I Patient's Demographics, Malignancy Characteristics and Chemotherapy Regimens of the Selected Population

\begin{tabular}{|c|c|c|c|c|}
\hline Variable & Total $(n=80)$ & Monocytopenia $(n=34)$ & Non-Monocytopenia $(n=46)$ & P-value \\
\hline Age (Years) & $36.5(42.5)$ & $26.5(45.5)$ & $41.0(36.0)$ & 0.355 \\
\hline Sex & & & & 0.049 \\
\hline Male & $38.0(47.5)$ & $21.0(61.8)$ & $17.0(37.0)$ & \\
\hline Female & $42.0(52.5)$ & $13.0(38.2)$ & $29.0(63.0)$ & \\
\hline Malignancy & & & & 0.317 \\
\hline Hematological & $36.0(45.0)$ & $18.0(52.9)$ & $18.0(38.3)$ & \\
\hline Solid & $44.0(55.0)$ & $16.0(47.1)$ & $28.0(60.9)$ & \\
\hline Distant metastasis & & & & 0.587 \\
\hline Yes & $17.0(21.3)$ & $6.00(17.6)$ & II.0 (23.9) & \\
\hline No & $63.0(78.8)$ & $28.0(82.4)$ & $35.0(76.1)$ & \\
\hline Chemotherapy cycles & $3.00(3.00)$ & $2.00(3.00)$ & $3.00(3.00)$ & 0.374 \\
\hline Co-morbidities $^{\mathrm{a}}$ & & & & 0.196 \\
\hline None & $59.0(73.8)$ & $23.0(67.6)$ & $36.0(78.3)$ & \\
\hline One Co-morbidity & $13.0(16.3)$ & $5.00(14.7)$ & $8.00(17.4)$ & \\
\hline Two Co-morbidities & $6.00(7.50)$ & $5.00(14.7)$ & $1.00(2.20)$ & \\
\hline Three Co-morbidities & $2.00(2.50)$ & $1.00(2.90)$ & $1.00(2.20)$ & \\
\hline Bone marrow involvement & & & & 0.111 \\
\hline Yes & $12.0(15.0)$ & $8.00(23.5)$ & $4.00(8.7)$ & \\
\hline No & $68.0(85.0)$ & $26.0(76.5)$ & $42.0(91.3)$ & \\
\hline Temperature at admission & $38.5(0.950)$ & $38.4(0.875)$ & $38.5(1.00)$ & 0.579 \\
\hline Presentation & & & & 0.780 \\
\hline Fever only & $46.0(57.5)$ & $21.0(6 \mathrm{I} .8)$ & $25.0(54.3)$ & \\
\hline Fever with cough & $10.0(12.5)$ & $3.00(8.80)$ & $7.00(15.2)$ & \\
\hline Fever with General weakness & $6.00(7.50)$ & $2.00(5.90)$ & $4.00(8.70)$ & \\
\hline Others $^{\mathrm{b}}$ & $18.0(22.5)$ & $8.00(23.5)$ & $10.0(21.7)$ & \\
\hline Chemotherapy regimens & & & & 0.218 \\
\hline ABVD & $5.00(6.30)$ & - & $5.00(10.9)$ & \\
\hline$A C$ & $5.00(6.30)$ & $1.00(2.90)$ & $4.00(8.70)$ & \\
\hline Hyper-CVAD & $3.00(3.80)$ & $2.00(5.90)$ & $1.00(2.20)$ & \\
\hline ICE & $4.00(5.00)$ & $1.00(2.90)$ & $3.00(6.50)$ & \\
\hline PACLITAXEL & $3.00(3.80)$ & $1.00(2.90)$ & $2.00(4.30)$ & \\
\hline $\mathrm{R}-\mathrm{CHOP}$ & $12.0(15.0)$ & $5.00(14.7)$ & $7.00(15.2)$ & \\
\hline R-ICE & $3.00(3.80)$ & $1.00(2.90)$ & $2.00(4.30)$ & \\
\hline VCD & $4.00(5.00)$ & $1.00(2.90)$ & $3.00(6.50)$ & \\
\hline Others ${ }^{c}$ & $41.0(51.3)$ & $22.0(64.7)$ & $19.0(4 \mid .3)$ & \\
\hline
\end{tabular}

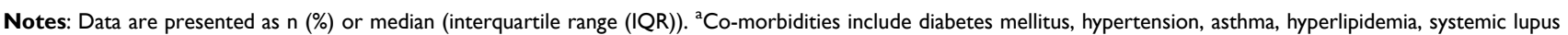
erythematosus, hypothyroidism, and hyperthyroidism. ${ }^{\text {} O t h e r ~ p r e s e n t a t i o n s ~ i n c l u d e ~ i r r i t a b i l i t y, ~ h y p o a c t i v i t y, ~ a b d o m i n a l ~ p a i n, ~ m e n o r r h a g i a, ~ b o n e ~ p a i n, ~ n i g h t ~ s w e a t s, ~ s k i n ~ r a s h, ~}$ diarrhea, and headache. 'Other chemotherapeutic regimens are discussed in the supplementary material (Table SI).

Abbreviations: ABVD, adriamycin, bleomycin, vinblastine, and dacarbazine; AC, adriamycin, and cyclophosphamide; Hyper-CVAD, hyperfractionated cyclophosphamide, vincristine, doxorubicin, and dexamethasone; ICE, ifosfamide, carboplatin, and etoposide; R-CHOP, rituximab, cyclophosphamide, daunorubicin, vincristine, and prednisone; R-ICE, rituximab, ifosfamide, carboplatin, and etoposide; VCD, bortezomib, cyclophosphamide, and dexamethasone.

$\left(\mathrm{F}(1,62)=60.157, P\right.$-value $\left.<0.001, \mathrm{R}^{2}=0.492\right)(\beta$ $=0.964, P$-value $<0.001)$, day four $(\mathrm{F}(1,44)=21.441$, $P$-value $\left.<0.001, \mathrm{R}^{2}=0.328\right)(\beta=0.786, P$-value $<$ $0.001)$, day five $\left(\mathrm{F}(1,30)=7.909, P\right.$-value $=0.009, \mathrm{R}^{2}=$ $0.209)(\beta=0.511, P$-value $=0.009)$ and finally, day six $(\mathrm{F}$
$(1,18)=12.969, P$-value $\left.=0.002, \mathrm{R}^{2}=0.647\right)(\beta=0.799$, $P$-value $=0.002$ ).

ROC analysis was used to evaluate the predictive role of $\mathrm{AMC}$ at admission regarding the ANC improvement after G-CSF therapy at different time intervals (Figure 3). A cutoff 
Table 2 Neutrophil Percentage, Absolute Neutrophil Count, and Absolute Neutrophil Count Difference at Several Days Following the Febrile Neutropenia Attack Treated with G-CSF

\begin{tabular}{|c|c|c|c|c|}
\hline Variable & Total $(n=80)$ & Monocytopenia $(n=34)$ & Non-Monocytopenia $(n=46)$ & P-value \\
\hline \multicolumn{5}{|l|}{ Admission } \\
\hline Neutrophils (\%) & $18.2 \pm 15.1$ & $17.7 \pm 17.5$ & $18.5 \pm 13.4$ & 0.819 \\
\hline ANC & $234 \pm 296$ & $186 \pm 333$ & $269 \pm 264$ & 0.215 \\
\hline ANC difference & - & - & - & - \\
\hline \multicolumn{5}{|l|}{ Day one } \\
\hline Neutrophils (\%) & $23.1 \pm 20.7$ & $16.0 \pm 15.0$ & $28.9 \pm 23.0$ & 0.011 \\
\hline ANC & $731.0 \pm 1438.0$ & $190.0 \pm 312.0$ & $1168.0 \pm 1810.0$ & 0.003 \\
\hline ANC difference & $61.2(290)$ & $3.3(106)$ & $145(6 \mid 3)$ & 0.006 \\
\hline \multicolumn{5}{|l|}{ Day two } \\
\hline Neutrophils (\%) & $40.2 \pm 26.0$ & $25.7 \pm 22.0$ & $51.4 \pm 23.3$ & $<0.001$ \\
\hline ANC & $2892.0 \pm 4288.0$ & $630.0 \pm 1237.0$ & $4640.0 \pm 4958.0$ & $<0.001$ \\
\hline ANC difference & $785(346 I)$ & $34.8(4 \mid 2)$ & $297 \mid(5333)$ & $<0.001$ \\
\hline \multicolumn{5}{|l|}{ Day three } \\
\hline Neutrophils (\%) & $49.7 \pm 28.8$ & $35.5 \pm 27.5$ & $63.9 \pm 22.5$ & $<0.001$ \\
\hline ANC & $288 I(97 \mid 7)$ & $446(2308)$ & $8973(12,257)$ & $<0.001$ \\
\hline ANC difference & $2779(9676)$ & $221(2242)$ & $8412(12,129)$ & $<0.001$ \\
\hline \multicolumn{5}{|l|}{ Day four } \\
\hline Neutrophils (\%) & $55.0 \pm 29.1$ & $47.4 \pm 30.1$ & $68.0 \pm 22.5$ & 0.012 \\
\hline ANC & $7078.0 \pm 8103.0$ & $4364.0 \pm 6633.0$ & $11,708.0 \pm 8459.0$ & 0.002 \\
\hline ANC difference & $3858(11,433)$ & $984(5376)$ & $10,883(|2,09|)$ & 0.001 \\
\hline \multicolumn{5}{|l|}{ Day five } \\
\hline Neutrophils (\%) & $53.8 \pm 28.1$ & $47.3 \pm 29.9$ & $68.1 \pm 17.6$ & 0.021 \\
\hline ANC & $2887(9105)$ & $1605(5325)$ & $8916(7100)$ & 0.035 \\
\hline ANC difference & $2875(9058)$ & |44| (5359) & $8755(6819)$ & 0.056 \\
\hline \multicolumn{5}{|l|}{ Day six } \\
\hline Neutrophils (\%) & $59.4 \pm 27.6$ & $51.3 \pm 30.6$ & $74.3 \pm 11.4$ & 0.028 \\
\hline ANC & $6565.0 \pm 6070.0$ & $4415.0 \pm 5158.0$ & $10,559.0 \pm 5901.0$ & 0.026 \\
\hline ANC difference & $3802.0(9931)$ & $1390(7856)$ & $11,010(12,073)$ & 0.019 \\
\hline Hospitalization period & $5.0(3.00)$ & $6.0(2.25)$ & $5.0(3.00)$ & 0.006 \\
\hline
\end{tabular}

Notes: Data is presented as mean \pm standard deviation (SD) or median (interquartile range (IQR)). ANC difference represents the difference between ANC at day (I-6) compared to the ANC at the onset of the febrile neutropenia attack.

value of 107 for ANC difference at day one yielded a sensitivity of $63.6 \%$ and a specificity of $65.2 \%$, with an AUC of 0.625. At the second day, a cutoff value of 103 for ANC difference yielded a sensitivity of $61.9 \%$ and a specificity of $73.3 \%$, with an AUC of 0.705 . At the third day, a cutoff value of 67.0 yielded a sensitivity of $64.3 \%$ and a specificity of $87.5 \%$, with an AUC of 0.752 . At the fourth day, a cutoff value of 17.4 yielded a sensitivity of $80.5 \%$ and a specificity of $60.0 \%$, with an AUC of 0.634 . At the fifth day, a cutoff value of 17.4 yielded a sensitivity of $67.9 \%$ and a specificity of $50.0 \%$, with an AUC of 0.518 . Finally, at the sixth day, a cutoff value of 3.2 yielded a sensitivity of $89.5 \%$ and a specificity of $100 \%$, with an AUC of 0.895 .
Kaplan-Meier estimator was used to assess the role of monocytopenia at the first attack in the recurrence probability of a second febrile neutropenia incidence. A total of 32 patients developed a second attack during the retrospective study period with 16 patients in each group. The Log rank test revealed no statistically significant difference between the monocytopenia and non- monocytopenia groups with $P$-value of 0.297 (Figure 4A). The same analysis was also applied to patients's mortality rates. In which 19 deaths in both groups, 8 in the nonmonocytopenia group and 11 in the monocytopenia group with no statistically significant difference (P-value $=0.198)$ as presented in Figure 4B. 


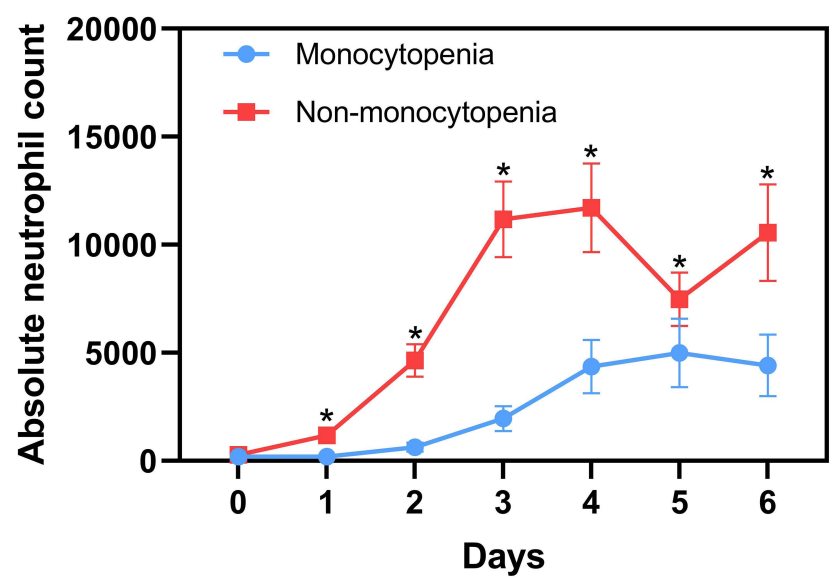

Figure I The short-term progression of patients with chemotherapy-induced febrile neutropenia $(n=80)$ and subsequently treated with G-CSF illustrated by the daily change in ANC values. Patients were categorized into two groups namely monocytopenia $(n=34)$ and non-monocytopenia $(n=46)$ groups with a cutoff point of $0.1 \times 10^{9}$ cells $/ \mathrm{L}$. Data points are presented as mean \pm standard error of the mean (SEM). *P-value $\leq 0.05$

\section{Discussion}

Chemotherapy-induced febrile neutropenia is a common and serious oncological emergency which develops in about $1 \%$ of patients undergoing a chemotherapeutic regimen and can reach up to $18 \%$ in the case of myelosuppressive agents' usage. $^{21-23}$ Certain patients have been identified to have a greater risk of developing febrile neutropenia, the components effecting such probability are treatment protocols, type of malignancy, and patient's demographics and characteristics. ${ }^{24}$ Age has been identified as one of the risk factors for patients with nonHodgkin lymphoma, in which it was noteworthy in patients older than 65 years. ${ }^{25-27}$ Female sex has been perceived as a potential risk factor in small-cell lung cancer patients who were more likely to be hospitalized, nevertheless sex was not identified as a significant risk factor in most studies. ${ }^{27,28}$ Multiple comorbidities have also been recognized as risk factors of developing febrile neutropenia including cardiovascular and renal diseases. All things considered, the number of comorbidities present concurrently with a malignancy type contribute to the hospitalization and mortality in neutropenic hospitalized patients. ${ }^{24}$ Certain chemotherapeutic agents are more prescient in inducing neutropenia including anthracyclines, vinorelbine, alkylators, taxanes, gemcitabine and topoisomerase inhibitors. In addition to the history of previous chemotherapy cycles and admission of three or more agents, notwithstanding the tumor type and stage. ${ }^{24}$ Genetic related risk factors including TP53 R72P and MDM2 SNP309, GSTM1 and UGTIA1 genotypes in patients treated by the FEC (fluorouracil + epirubicin + cyclophosphamide), FOLFOX (fluorouracil + oxaliplatin) and IROX (irinotecan + oxaliplatin) chemotherapy protocols respectively. ${ }^{29,30}$

As a result of the wide variation of risk factors, multiple risk indices have been developed including the Multinational Association for Supportive Care in Cancer (MASCC) and the Clinical Index of Stable Febrile Neutropenia (CISNE) guiding the clinical decision process. The MASCC index, which is now being widely used, was developed to identify patients with a lower risk of developing serious complications having a score of more than or equal to $21 .{ }^{1,31}$ Those patients are eligible for oral antibiotics treatment in the outpatient setting if being stable after 24 hours of monitoring. ${ }^{16}$ On the other hand, the CISNE index is a promising prognostic tool which divides patients into three categories depending on six variables including low ( 0 points), intermediate (1-2
A

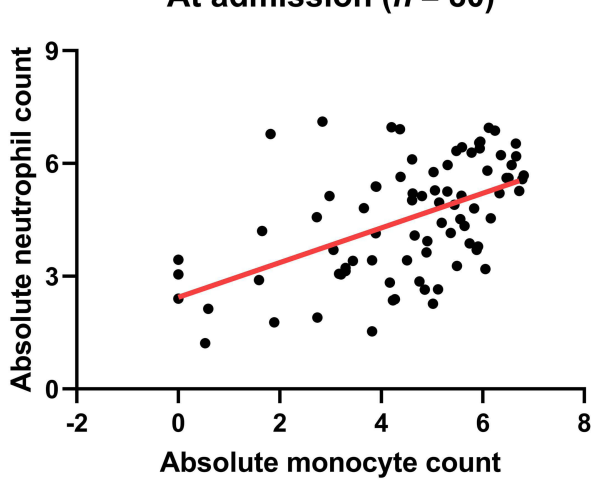

B

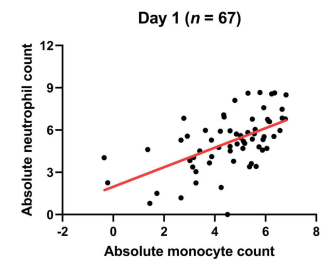

Day $4(n=46)$

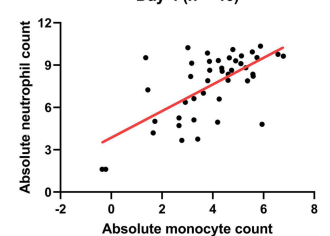

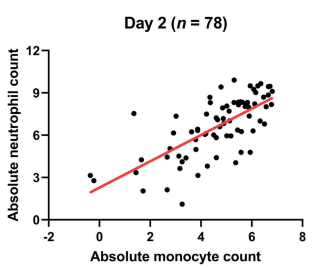

Day $5(n=32)$

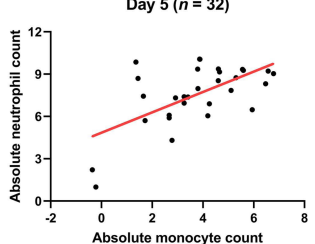

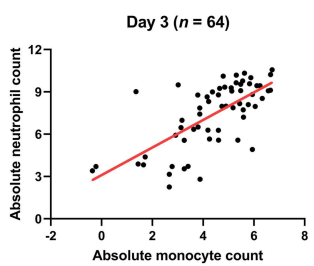

Day $6(n=20)$

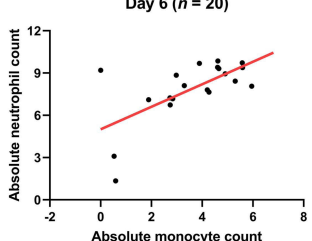

Figure 2 Linear regression analysis evaluated the association between AMC values at admission and ANC values at admission (A) and subsequent follow up days (B). AMC and ANC values were transformed using the natural logarithm $(\mathrm{In})$. 

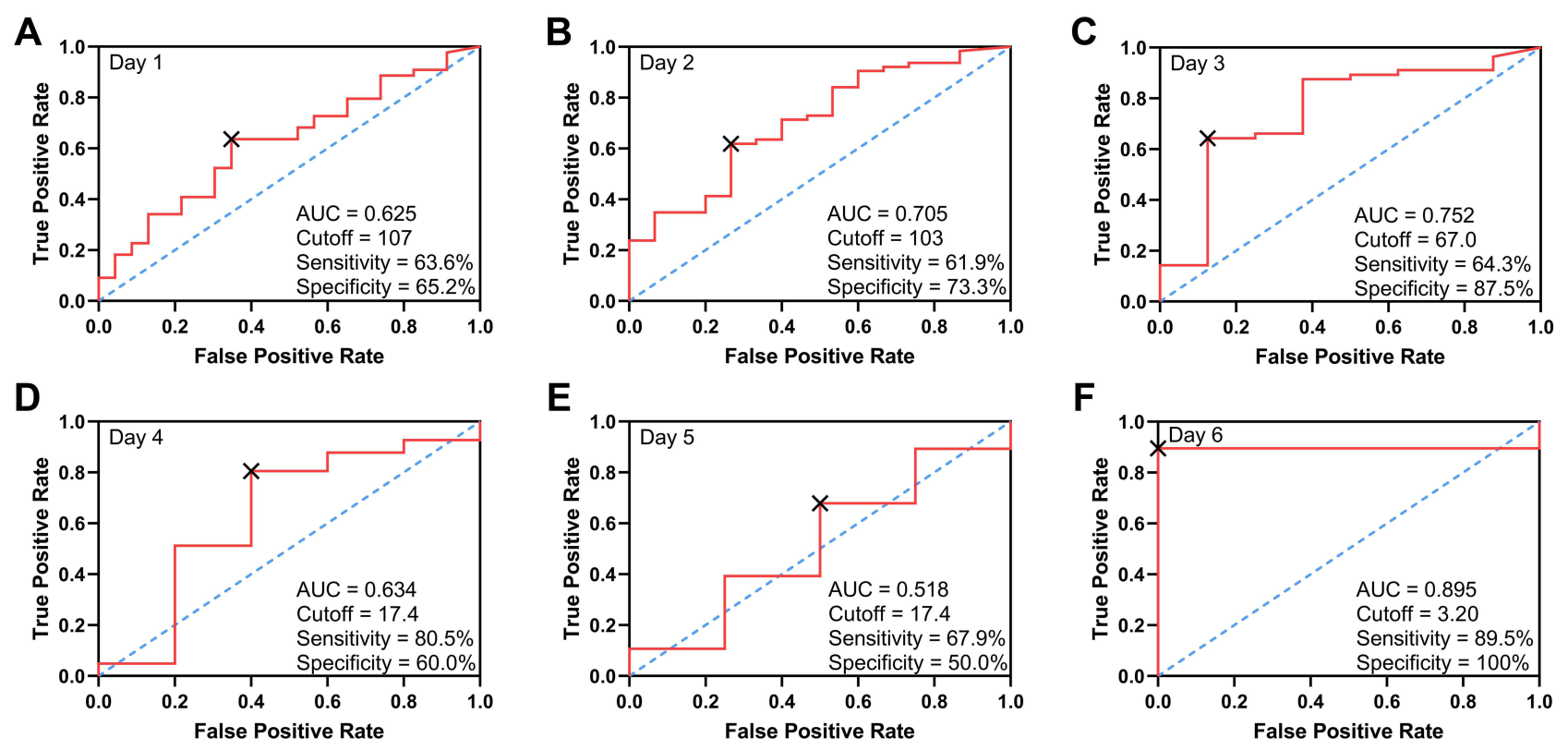

Figure 3 Receiver operating characteristic (ROC) curves of the AMC values at admission for prediction of ANC changes at day I to 6 (A-F).
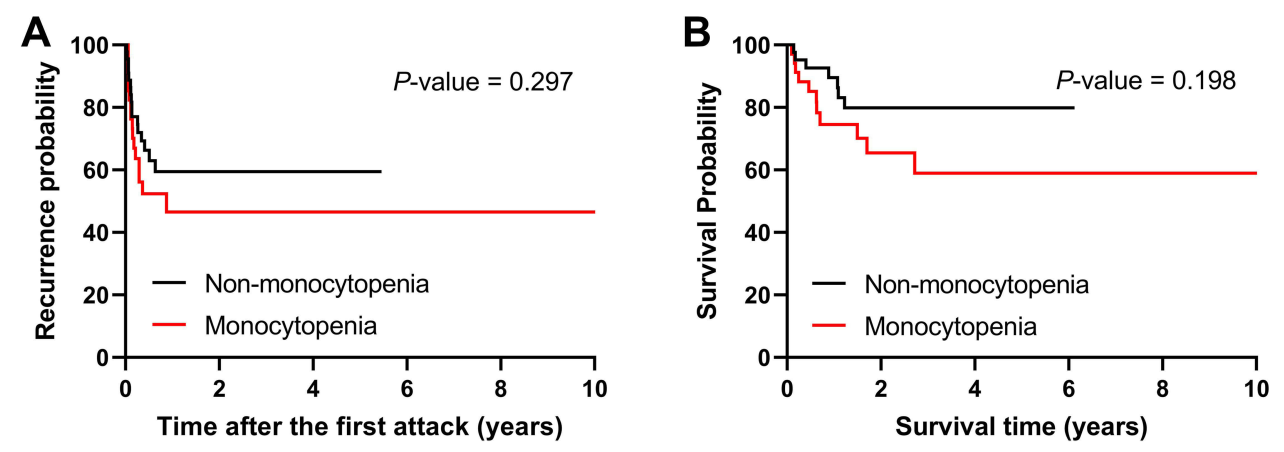

Figure 4 Kaplan-Meier curves to assess the role of monocytopenia in determining the recurrence (A) and survival (B) probability.

points), and high risk (3 points or more) patients for predicting critical complications in patients with solid tumors and stable episodes of febrile neutropenia. ${ }^{32}$ More recent evidence reveals that CISNE index can be more accurate and predictive than the MASCC score. ${ }^{33,34}$

A growing body of literature has been devoted to study the correlation between monocytes measures and neutropenia in several clinical settings. In a retrospective clinical study conducted by Moriyama et al, the utilization of monocyte nadir has been investigated as a clinical indicator for neutrophil nadir in patients with lung cancer who developed chemotherapy induced neutropenia. A strong correlation between ANC nadir and AMC nadir has been observed, which potentiates the role of AMC nadir in the prediction of ANC nadir and its timing. ${ }^{35}$ In another similar study, Shimanuki et al have investigated the use of pre- treatment hematological laboratory parameters to construct a prediction model for chemotherapy induced febrile neutropenia in patients with head and neck squamous cell carcinoma. The model has revealed that AMC values less than 370 cells $/ \mathrm{mm}^{3}$ are a good predictor for TPF (Docetaxel, cisplatin, and fluorouracil) regimen induced febrile neutropenia. ${ }^{36}$ Sato et al have studied the use of pretreatment monocytes percentage in the prediction of docetaxel monotherapy induced neutropenia in patients suffering from several types of malignancies. The study has demonstrated an inverse correlation between monocytes percentage and neutrophil count decrease in G-CSF administration and non-treated groups. ${ }^{37}$ Adding to this, Ouyang et al have demonstrated that monocytes and neutrophils count change possess the same time-wise trend in patients who developed chemotherapy induced neutropenia. In the same study, 
monocytes nadir occurred before neutrophil nadir with an inverse correlation between neutrophil decrease value and monocyte baseline percentage. ${ }^{38}$ Furthermore, a baseline monocyte count less than 150 cells per $\mu \mathrm{L}$ has been established as a bad prognostic variable in chemotherapy induced febrile neutropenia patients along with chemotherapeutic protocol, underlying malignancy, body surface area of less than or equal to $2 \mathrm{~m}^{2}$ and bone marrow involvement. ${ }^{39}$ In a retrospective study conducted among 62 patients with chemotherapy-induced febrile neutropenia, AMC and MP were evaluated for their short-term prognostic role regarding ANC changes, which revealed that MP can predict ANC changes with a cutoff point of $6.5 \%$ elucidating a sensitivity and specificity of 80 and $88.6 \%$ respectively and an AUC value of $0.908 .{ }^{20}$ Finally, multiple reports suggests that monocyte measures could have a predictive role in the clinical prognostic parameters of chemotherapyinduced febrile neutropenia. ${ }^{40,41}$

The exact pathophysiological mechanism that links $\mathrm{AMC}$ to ANC in the case of febrile neutropenia is not well understood or studied, which indicates the need to explore the cellular and molecular interactions in such clinical setting. The counter effects neutrophils and monocytes exhibit could be explained by hematopoiesis since they share a common progenitor namely "granulocytemonocyte progenitor". The differentiation route of neutrophils depends on the expression of C/EBP- $\varepsilon$ and Gfi-1 followed GATA-1 down expression, while C/EBP- $\alpha$ and PU.1 induce monocyte and macrophage generation. ${ }^{42}$ In addition, human adherent monocytes have experimentally illustrated a synthetic capability of hematopoiesis modulators such as G-CSF and GM-CSF. ${ }^{43}$ G-CSF is the main granulopoietic cytokine involved in neutrophil production and mobilization, it increases the survival of neutrophils, influences its function in infection sites and sensitize them to chemotactic factors. ${ }^{44,45}$ On the other hand, GM-CSF mediates neutrophil progenitor's proliferation and growth, other functionalities include its effect on mature neutrophils and the synergistic role with growth factors it exhibits. ${ }^{46,47}$ Monocytes even cause G-CSF driven mobilization of hematopoietic stem cells. ${ }^{44}$ So clinically speaking, a high monocyte count could result in partially higher concentration of these mediators leading to neutrophil production which could prevent neutropenia. G-CSF affects a variety of cellular pathways and modulates the secretory cytokine profile. ${ }^{48}$ These actions could influence monocytes to mediate granulopoiesis since they express the G-CSF receptor. ${ }^{49}$ Adding to this, G-CSF has been proven experimentally to modulate monocyte cytokine secretion. ${ }^{50,51}$ The exact mechanism of action should be subjected to further studies since such clinical observation has the potential to furnish a new insight in the fields of immunology and molecular biology along with its clinical applications.

\section{Conclusion}

In the current work, we represent a comprehensive analysis evaluating AMC values as a prognostic variable in determining ANC changes following G-CSF therapy in febrile neutropenia patients. A retrospective search throughout the electronic system of KAUH was performed representing a 10year single center data of febrile neutropenia cases. Based on the current analysis, AMC values hold a prognostic entity regarding ANC changes in chemotherapy-induced febrile neutropenia without any restriction to patients' demographics and characteristics following G-CSF treatment. The findings emphasize the role of AMC in notifying emergency doctors and oncology specialist toward high-risk febrile neutropenia patients and can predict the responsiveness of G-CSF therapy. The main limitation in this study is its retrospective nature with moderate sample size. The first attack of each patient was included, and the rest were omitted to eliminate the factor of response bias, which necessitate the need for additional analysis of recurrent attacks with their compatibility to the first one. Although AMC values could predict ANC changes to a certain degree, it did not exhibit any statistically significant difference in determining the morality and recurrence probability paths according to Kaplan-Meier analysis. Further studies are needed to increase the sample size and to specify the target population regarding the type of malignancy and chemotherapy protocol utilized. Future work should be also directed toward a more specific and sensitive risk prediction indices for chemotherapy-induced febrile neutropenia following G-CSF therapy.

\section{Ethical Approval}

The study was approved by the institutional review board committee in King Abdullah University Hospital (KAUH). This study was conducted in accordance with the declaration of Helsinki, applying all the current regulations for retrospective studies in KAUH. Patient consents were waived as data was used in aggregate anonymously.

\section{Author Contributions}

All authors made a significant contribution to the work reported, whether that is in the conception, study design, 
execution, acquisition of data, analysis, and interpretation, or in all these areas; took part in drafting, revising or critically reviewing the article; gave final approval of the version to be published; have agreed on the journal to which the article has been submitted; and agree to be accountable for all aspects of the work.

\section{Funding}

There is no funding to report.

\section{Disclosure}

The authors declare no conflicts of interest for this work.

\section{References}

1. Lucas AJ, Olin JL, Coleman MD. Management and preventive measures for febrile neutropenia. $P \&$ T. 2018;43(4):228-232.

2. Taplitz RA, Kennedy EB, Bow EJ, et al. Outpatient management of fever and neutropenia in adults treated for malignancy: American society of clinical oncology and infectious diseases society of America clinical practice guideline update. J Clin Oncol. 2018;36 (14):1443-1453. doi:10.1200/JCO.2017.77.6211

3. Klastersky J, de Naurois J, Rolston K, et al. Management of febrile neutropaenia: ESMO clinical practice guidelines广. Ann Oncol. 2016;27:v111-v118. doi:10.1093/annonc/mdw325

4. van der Velden WJFM, Herbers AHE, Netea MG, et al. Mucosal barrier injury, fever and infection in neutropenic patients with cancer: introducing the paradigm febrile mucositis. Br J Haematol. 2014;167 (4):441-452. doi:10.1111/bjh.13113

5. Netea MG, Kullberg BJ, Blok WL, et al. The role of hyperuricemia in the increased cytokine production after lipopolysaccharide challenge in neutropenic mice. Blood. 1997;89(2):577-582. doi:10.1182/blood. V89.2.577

6. Schwartzberg LS. Neutropenia: etiology and pathogenesis. Clin Cornerstone. 2006;8:S5-S11. doi:10.1016/S1098-3597(06)80053-0

7. Pagano L, Caira M, Nosari A, et al. Etiology of febrile episodes in patients with acute myeloid leukemia: results from the hema e-chart registry. Arch Intern Med. 2011;171(16):1502-1503. doi:10.1001/ archinternmed.2011.374

8. Weycker D, Li X, Edelsberg J, et al. Risk and consequences of chemotherapy-induced febrile neutropenia in patients with metastatic solid tumors. J Oncol Pract. 2015;11(1):47-54.

9. Weycker D, Barron R, Kartashov A, et al. Incidence, treatment, and consequences of chemotherapy-induced febrile neutropenia in the inpatient and outpatient settings. J Oncol Pharm Pract. 2014;20 (3):190-198. doi:10.1177/1078155213492450

10. Kanamaru A, Tatsumi Y. Microbiological data for patients with febrile neutropenia. Clin Infect Dis. 2004;39(Supplement_1):S7S10. doi: $10.1086 / 383042$

11. Freifeld AG, Bow EJ, Sepkowitz KA, et al. Clinical practice guideline for the use of antimicrobial agents in neutropenic patients with cancer: 2010 update by the Infectious Diseases Society of America. Clin Infect Dis. 2011;52(4):e56-e93.

12. Clark OA, Lyman GH, Castro AA, et al. Colony-stimulating factors for chemotherapy-induced febrile neutropenia: a meta-analysis of randomized controlled trials. J Clin Oncol. 2005;23(18):4198-4214. doi:10.1200/JCO.2005.05.645

13. Clark OAC, Lyman GH, Castro AA, et al. Colony-stimulating factors for chemotherapy-induced febrile neutropenia: a meta-analysis of randomized controlled trials. J Clin Oncol.. 2005;23(18):4198-4214.
14. Mhaskar R, Clark OA, Lyman G, et al. Colony-stimulating factors for chemotherapy-induced febrile neutropenia. Cochrane Database Syst Rev. 2014;10.

15. Brito-Zerón P, Soria N, Muñoz S, et al. Prevalence and clinical relevance of autoimmune neutropenia in patients with primary Sjögren's syndrome. Semin Arthritis Rheum. 2009;38(5):389-395. doi:10.1016/j.semarthrit.2008.01.014

16. Stewart DJ, Bodey GP. Infections in hairy cell leukemia (leukemic reticuloendotheliosis). Cancer. 1981;47(4):801-805. doi:10.1002/ 1097-0142(19810215)47:4<801::AID-CNCR2820470428>3.0.CO;2-6

17. Ogimi C, Krantz EM, Golob JL, et al. Antibiotic exposure prior to respiratory viral infection is associated with progression to lower respiratory tract disease in allogeneic hematopoietic cell transplant recipients. Biol Blood Marrow Transplant. 2018;24(11):2293-2301. doi:10.1016/j.bbmt.2018.05.016

18. Gui C, Cheng Z, Cecil E, et al. Low peripheral blood leukocyte counts during radiation therapy for head and neck cancer predict distant relapse and dysphagia. Int $J$ Radiat Oncol Biol Phys. 2019;105(1):E421. doi:10.1016/j.ijrobp.2019.06.1530

19. Georgiadou SP, Sampsonas FL, Rice D, et al. Open-lung biopsy in patients with undiagnosed lung lesions referred at a tertiary cancer center is safe and reveals noncancerous, noninfectious entities as the most common diagnoses. Eur J Clin Microbiol Infect Dis. 2013;32 (1):101-105. doi:10.1007/s10096-012-1720-9

20. Zheng B, Huang Z, Huang Y, et al. Predictive value of monocytes and lymphocytes for short-term neutrophil changes in chemotherapy-induced severe neutropenia in solid tumors. Supportive Care Cancer. 2020;28 (3):1289-1294. doi:10.1007/s00520-019-04946-3

21. Freifeld AG, Bow EJ, Sepkowitz KA, et al. Clinical practice guideline for the use of antimicrobial agents in neutropenic patients with cancer: 2010 update by the infectious diseases society of America. Clin Infect Dis. 2011;52(4):e56-e93.

22. Adelberg DE, Bishop MR. Emergencies related to cancer chemotherapy and hematopoietic stem cell transplantation. Emerg Med Clin North Am. 2009;27(2):311-331. doi:10.1016/j.emc.2009.01.005

23. Zheng B, Toarta C, Cheng W, et al. Accuracy of the Multinational Association of Supportive Care in Cancer (MASCC) and Clinical Index of Stable Febrile Neutropenia (CISNE) scores for predicting serious complications in adult patients with febrile neutropenia: a systematic review and meta-analysis. Crit Rev Oncol Hematol. 2020;149:102922.

24. Lyman GH, Abella E, Pettengell R. Risk factors for febrile neutropenia among patients with cancer receiving chemotherapy: a systematic review. Crit Rev Oncol Hematol. 2014;90(3):190-199. doi:10.1016/j.critrevonc.2013.12.006

25. Pettengell R, Bosly A, Szucs TD, et al. Multivariate analysis of febrile neutropenia occurrence in patients with non-Hodgkin lymphoma: data from the INC-EU Prospective Observational European Neutropenia Study. $B r \quad J \quad$ Haematol. 2009;144(5):677-685. doi:10.1111/j.1365-2141.2008.07514.x

26. Lyman GH, Morrison VA, Dale DC, et al. Risk of febrile neutropenia among patients with intermediate-grade non-Hodgkin's lymphoma receiving CHOP chemotherapy. Leuk Lymphoma. 2003;44 (12):2069-2076. doi:10.1080/1042819031000119262

27. Lyman GH, Delgado DJ. Risk and timing of hospitalization for febrile neutropenia in patients receiving CHOP, CHOP-R, or CNOP chemotherapy for intermediate-grade non-Hodgkin lymphoma. Cancer. 2003;98(11):2402-2409. doi:10.1002/cncr.11827

28. Crawford J, Glaspy JA, Stoller RG, et al. Final results of a placebo-controlled study of filgrastim in small-cell lung cancer: exploration of risk factors for febrile neutropenia. Support Cancer Ther. 2005;3(1):36-46. doi:10.3816/SCT.2005.n.023

29. Okishiro M, Kim SJ, Tsunashima R, et al. MDM2 SNP309 and TP53 $\mathrm{R} 72 \mathrm{P}$ associated with severe and febrile neutropenia in breast cancer patients treated with 5-FU/epirubicin/cyclophosphamide. Breast Cancer Res Treat. 2012;132(3):947-953. doi:10.1007/s10549-011$1637-5$ 
30. McLeod HL, Sargent DJ, Marsh S, et al. Pharmacogenetic predictors of adverse events and response to chemotherapy in metastatic colorectal cancer: results from north American gastrointestinal intergroup trial N9741. J Clin Oncol. 2010;28(20):3227-3233.

31. Klastersky J, Paesmans M, Rubenstein EB, et al. The multinational association for supportive care in cancer risk index: a multinational scoring system for identifying low-risk febrile neutropenic cancer patients. J Clin Oncol. 2000;18(16):3038-3051.

32. Carmona-Bayonas A, Gómez J, González-Billalabeitia E, et al. Prognostic evaluation of febrile neutropenia in apparently stable adult cancer patients. $\mathrm{Br} J$ Cancer. 2011;105(5):612-617. doi:10.1038/bjc.2011.284

33. Carmona-Bayonas A, Jiménez-Fonseca P, Virizuela Echaburu J, et al. Prediction of serious complications in patients with seemingly stable febrile neutropenia: validation of the clinical index of stable febrile neutropenia in a prospective cohort of patients from the FINITE study. J Clin Oncol. 2015;33(5):465-471.

34. Coyne CJ, Le V, Brennan JJ, et al. Application of the MASCC and CISNE risk-stratification scores to identify low-risk febrile neutropenic patients in the emergency department. Ann Emerg Med. 2017;69 (6):755-764. doi:10.1016/j.annemergmed.2016.11.007

35. Moriyama Y, Horita N, Kudo M, et al. Monocyte nadir is a possible indicator for neutrophil nadir during lung cancer chemotherapy. Clin Respir J. 2017;11(4):453-458. doi:10.1111/crj.12358

36. Shimanuki M, Imanishi Y, Sato Y, et al. Pretreatment monocyte counts and neutrophil counts predict the risk for febrile neutropenia in patients undergoing TPF chemotherapy for head and neck squamous cell carcinoma. Oncotarget. 2018;9(27):18970-18984. doi:10.18632/oncotarget.24863

37. Sato I, Nakaya N, Shimasaki T, Nakajima H, Motoo Y. Prediction of docetaxel monotherapy-induced neutropenia based on the monocyte percentage. Oncol Lett. 2012;3(4):860-864.

38. Ouyang W, Liu Y, Deng D, et al. The change in peripheral blood monocyte count: a predictor to make the management of chemotherapy-induced neutropenia. J Cancer Res Ther. 2018;14 (Supplement):S565-s570. doi:10.4103/0973-1482.177502

39. Moreau M, Klastersky J, Schwarzbold A, et al. A general chemotherapy myelotoxicity score to predict febrile neutropenia in hematological malignancies. Ann Oncol. 2009;20(3):513-519. doi:10.1093/ annonc/mdn655

40. Fonseca PJ, Carmona-Bayonas A, García IM, et al. A nomogram for predicting complications in patients with solid tumours and seemingly stable febrile neutropenia. $B r \quad J$ Cancer. 2016;114 (11):1191-1198. doi:10.1038/bjc.2016.118
41. Oguz A, Karadeniz C, Ckitak EC, et al. Which one is a risk factor for chemotherapy-induced febrile neutropenia in childhood solid tumors: early lymphopenia or monocytopenia? Pediatr Hematol Oncol. 2006;23(2):143-151. doi:10.1080/08880010500457673

42. Lawrence SM, Corriden R, Nizet V. The ontogeny of a neutrophil: mechanisms of granulopoiesis and homeostasis. Microbiol Mol Biol Rev. 2018;82(1):e00057-17. doi:10.1128/MMBR.00057-17

43. Sallerfors B, Olofsson T. Granulocyte-macrophage colony-stimulating factor (GM-CSF) and granulocyte colony-stimulating factor (G-CSF) secretion by adherent monocytes measured by quantitative immunoassays. Eur J Haematol. 1992;49 (4):199-207. doi:10.1111/j.1600-0609.1992.tb00047.x

44. Bendall LJ, Bradstock KF. G-CSF: from granulopoietic stimulant to bone marrow stem cell mobilizing agent. Cytokine Growth Factor Rev. 2014;25(4):355-367. doi:10.1016/j.cytogfr.2014.07.011

45. Castellani S, D'Oria S, Diana A, et al. G-CSF and GM-CSF modify neutrophil functions at concentrations found in cystic fibrosis. $\mathrm{Sci}$ Rep. 2019;9(1):12937. doi:10.1038/s41598-019-49419-z

46. Bober LA, Grace MJ, Pugliese-Sivo C, et al. The effect of GM-CSF and G-CSF on human neutrophil function. Immunopharmacology. 1995;29(2):111-119. doi:10.1016/0162-3109(94)00050-P

47. Fossati G, Mazzucchelli I, Gritti D, et al. In vitro effects of GM-CSF on mature peripheral blood neutrophils. Int J Mol Med. 1998;1 (6):943-951.

48. Rutella S, Zavala F, Danese S, et al. Granulocyte colony-stimulating factor: a novel mediator of $\mathrm{T}$ cell tolerance. J Immunol. 2005;175 (11):7085-7091. doi:10.4049/jimmunol.175.11.7085

49. Christopher MJ, Rao M, Liu F, et al. Expression of the G-CSF receptor in monocytic cells is sufficient to mediate hematopoietic progenitor mobilization by G-CSF in mice. J Exp Med. 2011;208 (2):251-260. doi:10.1084/jem.20101700

50. Saito M, Kiyokawa N, Taguchi T, et al. Granulocyte colony-stimulating factor directly affects human monocytes and modulates cytokine secretion. Exp Hematol. 2002;30(10):1115-1123. doi:10.1016/S0301-472X(02)00889-5

51. Cheng DE, Chang W-A, Hung J-Y, et al. Involvement of IL-10 and granulocyte colony-stimulating factor in the fate of monocytes controlled by galectin-1. Mol Med Rep. 2014;10(5):2389-2394. doi: $10.3892 / \mathrm{mmr} .2014 .2573$
Therapeutics and Clinical Risk Management

\section{Publish your work in this journal}

Therapeutics and Clinical Risk Management is an international, peerreviewed journal of clinical therapeutics and risk management, focusing on concise rapid reporting of clinical studies in all therapeutic areas, outcomes, safety, and programs for the effective, safe, and sustained use of medicines. This journal is indexed on PubMed Central, CAS,

\section{Dovepress}

EMBase, Scopus and the Elsevier Bibliographic databases. The manuscript management system is completely online and includes a very quick and fair peer-review system, which is all easy to use. Visit http://www.dovepress.com/testimonials.php to read real quotes from published authors. 